\title{
SOME BOUNDARY PROPERTIES OF THE RIEMANN MAPPING FUNCTION
}

\author{
MAYNARD G. ARSOVE ${ }^{1}$
}

1. Introduction. A function $\chi$ mapping the open unit disc $\omega$ conformally onto a simply connected proper subregion $\Omega$ of the complex plane will be called a Riemann mapping function for $\Omega$. Considerable attention has been devoted in the literature to the case when $\Omega$ is bounded by a Jordan curve, and the well-known Osgood-TaylorCarathéodory theorem asserts that $\chi$ can then be extended to a homeomorphism of $\bar{\omega}$ onto $\bar{\Omega}$. Recently this was generalized by showing that if $\Omega$ is bounded by any closed curve, then $\chi$ can be extended to a continuous mapping of $\bar{\omega}$ onto $\bar{\Omega}$, with $\partial \omega$ mapping onto $\partial \Omega$ (see [1] and [2]). Immediate applications are at hand, since a number of theorems stated for Jordan regions use only the continuous extensibility of $\chi$. This is the case, for example, with the theorems of Fejér and Lindelöf cited in $\$ 9$, Chapter IX, of [13]. It should be noted that the strengthened theorems represent a substantial improvement, since they apply to such fundamental regions as the disc slit along a radius.

Proceeding in a somewhat different direction, we propose to show how the generalized Osgood-Taylor-Carathéodory theorem can be used to improve certain fundamental formulas of classical analysis involving integrals along rectifiable boundary curves. Traditionally stated for Jordan boundary curves, these will be derived here for boundary curves which are not assumed simple. Other generalizations are also made, and the methods of proof are different from those previously given in the case of Jordan boundary curves.

To begin with, the generalized Osgood-Taylor-Carathéodory theorem enables us to characterize completely the regions $\Omega$ for which $\chi^{\prime}$ belongs to the Hardy class $H^{1}$.

THEOREM 1. Let $\Omega$ be a bounded simply connected plane region and $\chi$ a Riemann mapping function for $\Omega$. Then $\chi^{\prime}$ is in the Hardy class $H^{1}$ if and only if $\partial \Omega$ can be parametrized as a rectifiable closed curve.

The proof will be given in $\$ 2$. Although the "only if" part follows readily from a theorem of $F$. Riesz (see, e.g. [10, Chapter II, §5.7]), we include a short proof along different lines. Our applications are

Received by the editors August 31, 1966 and, in revised form, March 20, 1967.

1 Research supported by the National Science Foundation under grant GP 6181. 
based on the "if" part, which generalizes a classical theorem of $F$. and M. Riesz [13, p. 318].

Let us suppose initially that $\Omega$ is a bounded plane region for which $\partial \Omega$ can be parametrized as a closed curve. The continuous extensibility of the mapping function has the following implications: (i) the prime end boundary, or Carathéodory boundary, $\mathfrak{e}$ of $\Omega$ can be parametrized as a Jordan curve $\Lambda$, and (ii) each prime end is supported by a single Euclidean boundary point, so that $\Lambda$ traces out $\partial \Omega$ as a closed curve. We shall refer to $\Lambda$ as a Jordan-Carathéodory boundary curve for $\Omega$. Essentially the same situation prevails when $\Omega$ is a bounded plane region for which $\partial \Omega$ consists of a finite number $n$ of disjoint continua, each parametrizable as a closed curve. The Jordan-Carathêodory boundary then consists of $n$ Jordan-Carathéodory boundary curves corresponding to the components of $\partial \Omega$. These will be taken as oriented in the usual way.

For the remainder of this section let us adopt the blanket convention that $\Omega$ is given as a bounded plane region for which $\partial \Omega$ consists of finitely many disjoint continua, each parametrizable as a rectifiable closed curve, and $\Lambda$ designates the positively oriented Jordan-Carathéodory boundary of $\Omega$. Further, arc length will be denoted by $s$, Lebesgue plane measure by $a$, and the outward normal (wherever it exists) by $\nu$. Theorem 1 can be utilized in studying boundary properties for such regions, simply by looking at the boundary components one at a time. It follows that there exists a tangent (hence a normal) at almost all points of $\Lambda$ (see also [5]). At points where a normal exists it is always possible to insert a Stolz domain, and we can thus speak of nontangential approach to these points. ${ }^{2}$ Bounded harmonic (or analytic) functions admit nontangential limits almost everywhere on $\Lambda$, as can be seen by composing with a Riemann mapping function corresponding to a given component of $\partial \Omega$.

The above approach can be applied to obtain a strengthening of the so-called "stronger forms" of the Cauchy integral theorem and formula, and of Green's identities (see [4] and [8, Chapter X]). The latter lead, in turn, to a sharpening of earlier results on the absolute continuity of harmonic measure (see [12] and [14]).

Our conclusions can be stated as follows.

Theorem 2. For $f$ a bounded analytic function on $\Omega$, extended nontangentially, $z$ any point of $\Omega$, and $n=0,1, \cdots$ we have

2 It should be noted that, for more complicated boundaries, a generalized nontangential approach can be defined relative to Green's lines (see [3]). 


$$
\int_{\Lambda} f(\zeta) d \zeta=0 \quad \text { and } \quad f^{(n)}(z)=\frac{n !}{2 \pi i} \int_{\Lambda} \frac{f(\zeta)}{(\zeta-z)^{n+1}} d \zeta
$$

THEOREM 3. Let $u$ and $v$ be functions on $\Omega$ such that grad $u$ and grad $v$ exist as bounded continuous functions admitting nontangential limits almost everywhere on $\mathcal{C}$. Then $u$ and $v$ admit continuous extensions to $\Omega \cup \mathcal{C}$. For $u, v$ extended continuously and grad $u$, grad $v$ extended nontangentially the following formulas hold:

$$
\int_{\Omega} u \Delta v d a+\int_{\Omega}\left(u_{x} v_{x}+u_{y} v_{y}\right) d a=\int_{\Lambda} u \frac{\partial v}{\partial \nu} d s
$$

provided $v$ is $C^{\prime \prime}$ on $\Omega$ with $\Delta v$ summable;

$$
\int_{\Omega}(u \Delta v-v \Delta u) d a=\int_{\Lambda}\left(u \frac{\partial v}{\partial \nu}-v \frac{\partial u}{\partial \nu}\right) d s
$$

provided $u$ and $v$ are $C^{\prime \prime}$ on $\Omega$ with $\Delta u$ and $\Delta v$ summable.

Suppose further that $u[v]$ is harmonic, so that $\Delta u[\Delta v]$ drops out. Then (i) the existence of nontangential limits of grad $u$ [grad $v$ ] almost everywhere on $\mathrm{e}$ appears as a conclusion, rather than a hypothesis; and (ii) if $u[v]$ tends to a constant value on some component of $\partial \Omega$, then grad $u$ [grad $v$ ] need not be assumed bounded in the neighborhood of that component.

THEOREM 4. The harmonic measure $m_{z}$ for $\Omega$ at $z(\in \Omega)$, relative either to the Euclidean or Carathéodory boundary, is absolutely continuous with respect to arc length along $\Lambda$ and has linear density

$$
\frac{d m_{z}}{d s}=-\frac{1}{2 \pi} \frac{\partial G_{z}}{\partial \nu}
$$

where $G_{z}$ is the Green's function for $\Omega$ with pole at $z .^{3}$

Although the main proofs will be deferred until $\S 3$, we indicate here how Theorem 4 follows from Theorem 3 in the case of the Euclidean boundary. The conclusion (1.3) amounts just to the assertion that if $f$ is any continuous function on $\partial \Omega$, then the solution $H_{f}$ of the Dirichlet problem for $f$ on $\Omega$ is given by the expression

$$
H_{f}(z)=-\frac{1}{2 \pi} \int_{\Lambda} f \frac{\partial G_{z}}{\partial \nu} d s .
$$

\footnotetext{
${ }^{3}$ Since all boundary points lie in boundary continua, $\Omega$ is a Dirichlet region when compactified either by the Euclidean boundary or the Carathéodory boundary. The Green's functions are the same for the two compactifications.
} 
To establish (1.4), let us assume first that $f$ can be extended as a function $f^{*}$ of class $C^{(3)}$ on $\bar{\Omega}$, so that

$$
H_{f}(z)=f^{*}(z)+\frac{1}{2 \pi} \int_{\Omega} G_{z} \Delta f^{*} d a
$$

(see, e.g. [6, pp. 261-264]). Formula (1.2) can then be applied to the region $\Omega_{r}$ formed from $\Omega$ by punching out a closed disc of small radius $r$, the functions $u$ and $v$ being taken as $G_{z}$ and $f^{*}$, respectively. In the limit as $r \rightarrow 0$ the contribution due to the boundary of the disc reduces to $-2 \pi f^{*}(z)$, and the right-hand member in (1.5) therefore coincides with that in (1.4). The general case of arbitrary continuous $f$ then follows by extending $f$ continuously to the entire space and approximating the extended function uniformly over $\bar{\Omega}$ by functions of class $C^{(3)}$, for example, by forming triple areal means (see [11, pp. 10-11]). Note that the summability of $\partial G_{z} / \partial \nu$ over $\Lambda$, which is needed here, results at once from the choice of $f \equiv 1$ in (1.4).

2. The mapping theorem and some geometrical consequences. To prove Theorem 1, we start with the hypothesis that $\partial \Omega$ can be parametrized as a rectifiable closed curve and extend $\chi$ continuously to $\bar{\omega}$. The rest of the argument parallels the proof of the classical F. and M. Riesz theorem [13, pp. 318-319]. Defining $L_{n}(n=1,2, \cdots)$ on $\bar{\omega}$ as

$$
L_{n}(z)=\sum_{k=1}^{n}\left|\chi\left(z \exp 2 \pi i \frac{k-1}{n}\right)-\chi\left(z \exp 2 \pi i \frac{k}{n}\right)\right|,
$$

we see that each $L_{n}$ is subharmonic on $\omega$ and continuous on $\bar{\omega}$. It therefore attains a maximum at some point $z_{n}$ of $\partial \omega$, so that $L_{n}(r) \leqq L_{n}\left(z_{n}\right) \leqq s(\Lambda)$ for $0<r<1$. Hence,

$$
s\left(\Lambda^{r}\right) \leqq s(\Lambda),
$$

where $\Lambda^{r}$ is the curve $\chi\left(r e^{i \theta}\right)(0 \leqq \theta \leqq 2 \pi)$. On the other hand, the length of $\Lambda^{r}$ is given explicitly by the formula

$$
s\left(\Lambda^{r}\right)=\int_{0}^{2 \pi}\left|\chi^{\prime}\left(r e^{i \theta}\right)\right| r d \theta
$$

and (2.1) and (2.2) force $\chi^{\prime}$ to belong to the Hardy class $H^{1}$.

For the converse we assume $\chi^{\prime}$ to belong to the Hardy class $H^{1}$ and prove first that $\chi$ is uniformly continuous on $\omega$. If not, there will exist 
sequences $\left\{z_{n}\right\}$ and $\left\{z_{n}^{\prime}\right\}$ in $\omega$ converging to some point $z_{0}$ of $\partial \omega$ and such that for some $\epsilon>0$

$$
\left|\chi\left(z_{n}\right)-\chi\left(z_{n}^{\prime}\right)\right| \geqq \epsilon \quad(n=1,2, \cdots) .
$$

Standard properties of $H^{1}$ ensure that $\chi^{\prime}$ has nontangential limits at almost all points of $\partial \omega$, denoted in the usual way by $\chi^{\prime}\left(e^{i \theta}\right)$, and that

$$
\lim _{r \rightarrow 1} \int_{0}^{2 \pi}\left|\chi^{\prime}\left(r e^{i \theta}\right)\right| d \theta=\int_{0}^{2 \pi}\left|\chi^{\prime}\left(e^{i \theta}\right)\right| d \theta .
$$

This allows application of a theorem of Vitali (see the Introduction of $[10]$ or $[7, \mathrm{pp} .150,234])$ to the effect that the integral of $\left|\chi^{\prime}\left(r e^{i \theta}\right)\right| d \theta$ is uniformly absolutely continuous. We can thus find an open interval $I$ centered at $\arg z_{0}$ and a number $\rho(<1)$ such that

$$
\int_{I}\left|\chi^{\prime}\left(r e^{i \theta}\right)\right| d \theta<\frac{\epsilon}{3} \quad(\rho<r<1) .
$$

Now, there exists a radius segment $R$ with initial angle in $I$ and along which $\left|\chi^{\prime}\right|<M$ (=const), and we shall restrict $\rho$ so that

$$
1-\rho<\epsilon / 3 M \text {. }
$$

Taking $z_{n}$ and $z_{n}^{\prime}$ in the annular sector $\left\{r e^{i \theta}: \rho<r<1, \theta \in I\right\}$, we inte. grate $\left|\chi^{\prime}\right|$ along a path in this sector comprised of an arc joining $z_{n}$ to $R$, a segment along $R$, and an arc joining $R$ to $z_{n}{ }^{\prime}$. By (2.5) and (2.6) the value of this integral is less than $\epsilon$, contradicting (2.3). Uniform continuity of $\chi$ on $\omega$ is thus established.

This permits us to extend $\chi$ so as to be continuous on $\bar{\omega}$, and it follows that $\partial \Omega$ is parametrizable as a closed curve. Approximation by inscribed polygonal curves yields

$$
\liminf _{r \rightarrow 1} s\left(\Lambda^{r}\right) \geqq s(\Lambda),
$$

from which we infer, via (2.2), that $s(\Lambda)$ must be finite. Theorem 1 is now proved in full.

Let us indicate some further conclusions that can be drawn in the setting of Theorem 1. First, combining (2.1), (2.2), (2.4), and (2.7) results in

$$
s(\Lambda)=\lim _{r \rightarrow 1} \int_{0}^{2 \pi}\left|\chi^{\prime}\left(r e^{i \theta}\right)\right| d \theta=\int_{0}^{2 \pi}\left|\chi^{\prime}\left(e^{i \theta}\right)\right| d \theta .
$$

Exactly as in the proof of the classical F. and M. Riesz theorem [13, 
p. 319] this implies that $\chi\left(e^{i \theta}\right)$ is an absolutely continuous function of $\theta$ and that, for almost all $\theta$,

$$
\frac{d \chi\left(e^{i \theta}\right)}{d \theta}=i e^{i \theta} \chi^{\prime}\left(e^{i \theta}\right) \text { and } \frac{d s}{d \theta}=\left|\chi^{\prime}\left(e^{i \theta}\right)\right| .
$$

Moreover, a familiar property of the space $H^{1}$ ensures that

$$
\chi^{\prime}\left(r e^{i \theta}\right) \underset{r \rightarrow 1}{\longrightarrow} \chi^{\prime}\left(e^{i \theta}\right) \text { in } L^{1}
$$

(as functions of $\theta) .^{4}$

It is well known that $\chi^{\prime}\left(e^{i \theta}\right) \neq 0$ almost everywhere $([10$, p. 56] or $[13$, p. 319]). At such points the curve $\Lambda$ admits the tangent and normal vectors

$$
\tau=\frac{i e^{i \theta} \chi^{\prime}\left(e^{i \theta}\right)}{\left|\chi^{\prime}\left(e^{i \theta}\right)\right|} \quad \text { and } \quad \nu=\frac{e^{i \theta} \chi^{\prime}\left(e^{i \theta}\right)}{\left|\chi^{\prime}\left(e^{i \theta}\right)\right|},
$$

respectively, and $\Omega$ admits a Stolz domain with axis along the normal and arbitrary angular width $(<\pi)$. For the latter assertion it is convenient to observe that almost all radii in $\omega$ have images (Green's lines) terminating in points of $\partial \Omega$; these can be used to form a flux tube into which a Stol $z$ domain can be fitted. We note also that the mapping $\chi$ remains conformal at such points.

Suppose now that $u$ is a $C^{\prime}$ function on $\Omega$ for which grad $u$ has a finite nontangential limit at a point $\zeta$ of $\Lambda$ admitting a normal. By the mean-value theorem, $u$ is easily seen to be uniformly continuous on Stolz domains with vertex at $\zeta$, so that $u$ itself has a finite nontangential limit at $\zeta$. Another application of the mean-value theorem shows that $u$ has a normal derivative at $\zeta$ given by $u_{x} \cos \alpha+u_{y} \sin \alpha$, where $\alpha=\arg \nu$. Denoting the conjugate gradient operator by $\nabla$, i.e. $\nabla u=u_{x}-i u_{y}$, we can write this as

$$
\frac{\partial u}{\partial \nu}=\operatorname{Re}(\nu \nabla u)
$$

Formula (2.11) holds almost everywhere on $\Lambda$, and a corresponding formula holds on $\Lambda^{r}$.

3. Applications of the mapping theorem. For notational brevity the proof of Theorem 2 and the first half of Theorem 3 will be carried out only for $\Omega$ simply connected. The general case can then be de-

4 As pointed out by John V. Ryff, this leads at once to the absolute continuity of $\chi\left(e^{i \theta}\right)$, simply by passing to the limit in the expression $\chi\left(r e^{i \theta}\right)-\chi(r)=i r \int_{0}^{\theta} e^{i t} \chi^{\prime}\left(r e^{i t}\right) d t$. 
rived by using the same limiting procedures relative to each of the boundary curves (after performing an inversion in the case of inner boundary curves). We thus maintain the setting of $\$ 2$.

Proof of Theorem 2. Here $f$ is analytic on $\Omega$, so that

$$
0=\int_{\Lambda^{r}} f(\zeta) d \zeta=\int_{0}^{2 \pi} f\left[\chi\left(r e^{i \theta}\right)\right] \chi^{\prime}\left(r e^{i \theta}\right) i r e^{i \theta} d \theta .
$$

In terms of the nontangential extension of $f$ we also have

$$
\int_{\Lambda} f(\zeta) d \zeta=\int_{0}^{2 \pi} f\left[\chi\left(e^{i \theta}\right)\right] \chi^{\prime}\left(e^{i \theta}\right) i e^{i \theta} d \theta
$$

since the absolute continuity of $\chi\left(e^{i \theta}\right)$, in conjunction with (2.9), allows application of the change of variables theorem for LebesgueStieltjes integrals (see [9, p. 280]). To conclude that (3.2) vanishes, we have only to justify passing to the limit from (3.1) as $r \rightarrow 1$. This, however, is immediate from the $L^{1}$ convergence property in (2.10), coupled with the boundedness of the rest of the integrand. The second assertion in Theorem 2 can be established in precisely the same way, since $1 /(\zeta-z)^{n+1}$ is a continuous function of $\zeta(\neq z)$.

Proof of Theorem 3. The assertion that $u$ admits a continuous extension to $\Omega \cup \mathcal{C}$ is equivalent to the assertion that the composite function $u \circ \chi$ is uniformly continuous on $\omega$. This uniform continuity property can be proved by observing first that $\nabla(u \circ \chi)=[(\nabla u) \circ \chi] \cdot \chi^{\prime}$ and then using the argument employed in $\$ 2$ to establish uniform continuity of $\chi$. Hence, $u$ and $v$ admit continuous extensions to $\Omega \cup \mathfrak{C}$.

Along $\Lambda^{r}$ the line differential $(\partial v / \partial \nu) d s$ appears as

$$
\frac{\partial u}{\partial \nu} d s=\operatorname{Re}\left\{r e^{i \theta} \chi^{\prime}\left(r e^{i \theta}\right) \nabla v\left[\chi\left(r e^{i \theta}\right)\right]\right\} d \theta,
$$

in view of (2.11) and (2.9). Therefore

$$
\int_{\Lambda^{r}} u \frac{\partial v}{\partial \nu} d s=\int_{0}^{2 \pi} u\left[\chi\left(r e^{i \theta}\right)\right] \operatorname{Re}\left\{r e^{i \theta} \chi^{\prime}\left(r e^{i \theta}\right) \nabla v\left[\chi\left(r e^{i \theta}\right)\right]\right\}[d \theta .
$$

As in the case of analytic functions, this formula remains valid for $r=1$ provided we replace $\Lambda^{r}$ by $\Lambda$. Moreover, as $r \rightarrow 1$

$$
u\left[\chi\left(r e^{i \theta}\right)\right] \nabla v\left[\chi\left(r e^{i \theta}\right)\right] \rightarrow u\left[\chi\left(e^{i \theta}\right)\right] \nabla v\left[\chi\left(e^{i \theta}\right)\right]
$$

boundedly, and the $L^{1}$ convergence property (2.10) can be invoked once more to conclude that

$$
\lim _{r \rightarrow 1} \int_{\Lambda^{r}} u \frac{\partial v}{\partial \nu} d s=\int_{\Lambda} u \frac{\partial v}{\partial \nu} d s
$$


Now, Green's identity in its classical formulation holds over the region $\Omega^{r}$ enclosed by $\Lambda^{r}$, and we thus have

$$
\int_{\Omega^{r}} u \Delta v d a+\int_{\Omega^{r}}\left(u_{x} v_{x}+u_{y} v_{y}\right) d a=\int_{\Lambda^{r}} u \frac{\partial v}{\partial \nu} d s .
$$

To complete the derivation of formula (1.1) all that remains is to pass to the limit on $r$, taking account of (3.3) and the evident fact that the left-hand member here tends to the corresponding integral over $\Omega$. Formula (1.2) then follows by symmetry in the usual way.

The remark (ii) must still be substantiated, and for this it suffices to consider the case of a harmonic function $v$ tending to 0 on some component $B$ of $\partial \Omega$. Introducing a mapping $\chi$ corresponding to $B$, we observe that, since $v \circ \chi$ tends to 0 on $\partial \omega$, it can be continued harmonically across $\partial \omega$. Three conclusions ensue. First, grad $v$ has finite nontangential limits almost everywhere on $\mathfrak{C}$, in view of the identity $(\nabla v) \circ \chi=[\nabla(v \circ \chi)] / \chi^{\prime}$. Second, the limiting relation (3.3) holds, since now

$$
\chi^{\prime}\left(r e^{i \theta}\right) \nabla v\left[\chi\left(r e^{i \theta}\right)\right] \rightarrow \chi^{\prime}\left(e^{i \theta}\right) \nabla v\left[\chi\left(e^{i \theta}\right)\right]
$$

boundedly as $r \rightarrow 1$. Third, by conformal invariance, the Dirichlet integral of $v$ in the neighborhood of $B$ is finite, so that the passage to the limit in the left-hand member of (3.4) is justified.

Proof of Theorem 4 in the case of the Carathéodory boundARY. Here we start with a continuous function $f$ on $\mathfrak{e}$ and observe that there is no loss of generality in assuming $f$ to vanish on all but one component $B$ of $\partial \Omega$. A mapping $\chi$ corresponding to $B$ is then chosen so that $\chi(0)=z$. Clearly, $\chi^{-1}(\Omega)$ is a subregion $\omega^{*}$ of $\omega$ with the unit circle as its outer boundary and with Green's function $G_{0}^{*}$ $=G_{z} \circ \chi$. Since $G_{0}^{*}$ can be continued harmonically across $\partial \omega$ and $\nabla G_{0}^{*}=\left[\left(\nabla G_{z}\right) \circ \chi\right] \cdot \chi^{\prime}$, the Euclidean case already treated yields

$$
H_{f}(z)=-\frac{1}{2 \pi} \int_{0}^{2 \pi} f\left[\chi\left(e^{i \theta}\right)\right] \operatorname{Re}\left\{e^{i \theta} \chi^{\prime}\left(e^{i \theta}\right) \nabla G_{z}\left[\chi\left(e^{i \theta}\right)\right]\right\} d \theta .
$$

This, however, is just the desired formula (1.4).

\section{REFERENCES}

1. M. G. Arsove, The Osgood-Taylor-Carathéodory theorem, Proc. Amer. Math. Soc. 19 (1968), 38-44.

2. - Intrinsic characterization of regions bounded by closed curves, Duke Math. J. 34 (1967), 425-430.

3. M. G. Arsove and G. Johnson, Jr., A conformal mapping technique for infinitely connected regions (to appear). 
4. E. F. Beckenbach, The stronger form of Cauchy's integral theorem, Bull. Amer. Math. Soc. 49 (1943), 615-618.

5. A. S. Besicovitch, On the existence of tangents to rectifiable curves, J. London Math. Soc. 19 (1944), 205-207.

6. R. Courant and D. Hilbert, Methods of mathematical physics, Vol. II, Interscience, New York, 1962.

7. N. Dunford and J. T. Schwartz, Linear operators, Part I: General theory, Interscience, New York, 1958.

8. G. M. Golusin, Geometrische Funktionentheorie, VEB Deutscher Verlag, Berlin, 1957.

9. L. M. Graves, The theory of functions of real variables, 2nd ed., McGraw-Hill, New York, 1956.

10. I. I. Privalow, Randeigenschaften analytischer Funktionen, VEB Deutscher Verlag, Berlin, 1956.

11. T. Rad6, Subharmonic functions, Springer-Verlag, Berlin, 1937.

12. M. Tsuji, On the Green's function, Japan J. Math. 18 (1942), 379-383.

13. - Potential theory in modern function theory, Maruzen, Tokyo, 1959.

14. S. Verblunsky, On a fundamental formula of potential theory, J. London Math. Soc. 26 (1951), 25-30.

UNIVERSITY OF WASHINGTON 